\title{
Questionário de Sentido de Vida: Evidências de sua Validade Fatorial e Consistência Interna
}

Meaning in Life Questionnaire: Evidence of its Factor Validity

Cuestionario de Sentido de Vida: Evidencias de su Validez Factorial y Fiabilidad

Thiago Antonio Avellar de Aquino, Valdiney Gouveia Veloso, Andrei Alves de Aguiar, Gylmara de Araújo Pereira \& Ana Sandra Fernandes

Universidade Federal da Paraíba

Tiago Deividy Bento Serafim Faculdade Vale do Salgado

Alisson de Meneses Pontes

Faculdades Integradas de Patos

http://dx.doi.org/10.1590/1982-3703001332012 
Thiago Antonio Avellar de Aquino, Valdiney Gouveia Veloso, Andrei Alves de Aguiar, Tiago Deividy Bento Serafim, Alisson de Meneses Pontes, Gylmara de Araújo Pereira \& Ana Sandra Fernandes

Resumo: A presente pesquisa teve por objetivo adaptar o Questionário Sentido de Vida (QSV), reunindo evidências de sua validade fatorial e consistência interna. Realizaram-se dois estudos junto a estudantes universitários de João Pessoa, PB. No estudo 1 participaram 414 voluntários, a maioria do sexo feminino $(63,5 \%)$, com idade média de 28,2 anos $(d p=9,50)$. No estudo 2 participaram 201 pessoas, a maioria do sexo feminino (74,6\%), com idade média de 26,7 anos $(d p=9,56)$. Nos dois estudos os participantes responderam o QSV e perguntas demográficas, sendo que os do segundo responderam dois outros instrumentos: Teste Propósito de Vida (Pil-Teste) e Escala de Percepção Ontológica do Tempo (EPOT). No estudo 1 foi realizada uma análise fatorial confirmatória, sugerindo a adequação da estrutura bifatorial [GFI $=0,94, A G F I=0,90$, CFI = 0,95 e RMSEA =0,086]; os alfas de Cronbach dos fatores foram 0,89 (busca de sentido) e 0,85 (presença de sentido). No estudo 2 foi observada evidência de validade convergente do QSV com o Pil-Teste e a EPOT. Concluiu-se que o QSV tem demonstrado ser pertinente para avaliar, nos modos propostos, o sentido de vida, podendo ser utilizado em pesquisas no contexto brasileiro.

Palavras-chave: Sentido de vida. Validade. Adaptação.

Abstract: This research aimed to adapt the Meaning in Life Questionnaire (MLQ) gathering evidence of its factorial validity and reliability. Two studies were held with undergraduate students from Joao Pessoa, Paraiba state, Brazil. In Study 1, participants were 414 volunteers, mostly female $(63.5 \%)$, with a mean age of $28.2(s d=9.56)$. Study 2 involved 201 subjects, mostly female $(74.6 \%)$, with a mean age of $26.7(s d=9.50)$. The participants of both studies answered the MLQ and demographic questions. The participants of Study 2 also answered the following instruments: Purpose of Life Test (Pil-Test) and Ontological Time Perception Scale (OTPS). In Study 1 , a confirmatory factor analysis was performed, suggesting the appropriateness of the two-factor structure $[G F I=.94, A G F I=.90, C F I=.95$, and RMSEA $=0.086]$; the Cronbach's alphas of these factors were .89 (search for meaning) and .85 (presence of sense). In Study 2, we observed evidence of convergent validity of the MLQ with respect to the Pil-Test and OTPS. In conclusion, the $M L Q$ was considered an appropriate instrument to measure the life purpose in the way it was proposed, being useful in research in the Brazilian context.

Keywords: Meaning in life. Validity. Adaptation.

Resumen: La presente investigación tuvo como objetivo adaptar el Cuestionario Sentido de Vida (CSV), reuniendo evidencias de su validez factorial y fiabilidad. Se han llevado a cabo dos estudios con estudiantes universitarios de João Pessoa (PB). En el Estudio 1 han participado 414 voluntarios, la mayoría mujeres (63.5\%), con edad promedia de 28.2 años $(d t=9.50)$. En el Estudio 2 los participantes han sido 201 personas, la mayoría mujeres (74.6\%), con edad promedia de 26.7 años $(d t=9.56)$. En ambos estudios los participantes contestaron al CSV y preguntas demográficas, siendo que aquellos del Estudio 2 aún respondieron a otros dos instrumentos: el Teste Propósito de Vida (Pil-Test) y la Escala de Percepción Ontológica del Tiempo (EPOT). En el Estudio 1 se realizó un análisis factorial confirmatorio, sugiriendo la adecuación de la estructura con dos factores $[G F I=0.94, A G F I=0.90, C F I=0.95$ y RMSEA $=0.086]$; sus alfas de Cronbach fueron 0.89 (búsqueda de sentido) y 0.85 (presencia de sentido). En el Estudio 2 se observó evidencia de validez convergente del CSV con el Pil-Test y la EPOT. Se concluyó que el CSV se mostró adecuado para medir el sentido de vida, de la forma que ha sido propuesto, pudiendo utilizarse en investigaciones dentro del contexto brasileño.

Palabras-clave: Sentido de la vida. Validez. Adaptación.

\section{Introdução}

O sentido de vida é uma preocupação especificamente humana e constitui objeto do pensamento ao longo da história da Filosofia. Ademais, este construto tornou-se uma das temáticas relevantes em abordagens fenomenológicas e existenciais quando passou a ser um dos principais objetos de reflexão de filósofos da existência (Buber, 2001; Heidegger, 1989; Kierkegaard, 1976; Sartre, 1987). Posteriormente, com o advento da 
Thiago Antonio Avellar de Aquino, Valdiney Gouveia Veloso, Andrei Alves de Aguiar, Tiago Deividy Bento Serafim, Alisson de Meneses Pontes, Gylmara de Araújo Pereira \& Ana Sandra Fernandes

psiquiatria existencial, ele foi abordado em uma perspectiva clínica por diversos profissionais, a exemplo de Viktor Frankl (1989), Medarde Boss (1976) e Kal Jaspers (1973).

Na tentativa de compreender e descrever a busca do ser humano por um sentido na vida, o método fenomenológico foi o mais privilegiado (Husserl, 1965, 2006), norteando, inicialmente, as reflexões dos precursores da psicologia existencial. Uma vez que o método fenomenológico se detém na análise da consciência e da intencionalidade (Abbagnano, 1976), os estudos que o fundamentam apresentam um caráter mais subjetivo. Essa ênfase, por sua vez, retardou o surgimento de análises mais objetivas que pudessem reconhecer, por meio de métodos atuais, os conceitos da psicologia existencial.

Não obstante, alguns autores construíram e reuniram evidências de validade de tal construto (e.g., Crumbaugh \& Maholick, 1964; Lukas, 1988; Steger, Frazier, Oishi \& Kaler, 2006). Tendo em vista a relevância teórica e prática desse conceito, a presente pesquisa justifica-se na medida em que se constata um aumento de estudos empíricos para avaliar os pressupostos que fundamentam as concepções da psicologia e psiquiatria existenciais, especialmente na vertente da Logoterapia, que se constitui como uma análise existencial do sentido da vida.

\section{A questão do sentido da vida na Psicologia}

Embora existam outros autores que trataram sobre o sentido da vida (e.g., Baumeister, 1991), é evidente a contribuição do neurologista e psiquiatra Viktor Frankl. Considera-se que ele tenha sido um dos mais importantes teóricos a refletir de forma científica e filosófica sobre tal problemática, tendo publicado 39 livros a respeito. Frankl (1989) inaugurou uma escola de psicoterapia denominada Logoterapia e análise existencial, sendo mais conhecida como a psicoterapia centrada no sentido da vida, sucedida pela Psicanálise de Freud (1976) e a Psicologia Individual de Adler (1931/1998).

A Logoterapia é uma proposta de re-humanização das psicoterapias por meio dos seus três fundamentos: a liberdade da vontade, a vontade de sentido e o sentido da vida (Lukas, 1989). Tomando como base esses três princípios, Frankl (1989) construiu os fundamentos do seu existencialismo, constituindo uma teoria psicológica com uma profunda fundamentação filosófica. Procura-se resumi-los a seguir.

Liberdade da vontade. Frankl (1989) advoga que o ser humano pode vir a ser incondicionado, pois ele é um ser que escolhe. Desse modo, entende que ele não é apenas determinado, mas determina-se a si mesmo. Entretanto, de fato, sugere que o mesmo ser é condicionado tanto pela dimensão biológica quanto pelas dimensões psíquica e social, mas que também assume posturas perante aquilo que o condiciona. Em última instância, o ser humano não seria livre dos condicionamentos, mas livre para decidir-se perante eles.

Vontade de sentido. Constitui-se na motivação primária da existência humana e pode ser definida como "simplesmente aquilo que é frustrado no homem sempre que ele é tomado pelo sentimento de falta de sentido e de vazio" (Frankl, 1991, p. 25). Esse construto pode ser inferido empiricamente por meio de instrumentos psicométricos. Dessa forma, Frankl (2011) reconhece a legitimidade de uma "psicometria da vontade de sentido", que pode estar, de fato, associada a uma concepção determinista, mas que não necessariamente resultaria em uma postura pandeterminista, isto é, que o ser humano não teria qualquer possibilidade de escolha.

Sentido da vida. Esse é um axioma filosófico que concebe que, se há uma busca de sentido por parte do ser humano, há também um sentido a ser desvelado e o mesmo estaria latente nas situações (Frankl, 2008). Sobretudo o ser humano encontraria sentido na vida por meio de sua relação com o mundo, pois sua estrutura existencial se caracteriza como um "ser no mundo". Frankl (1989) propõe, derivada dessa concepção, uma tríade valorativa pela qual o ser humano realizaria sentidos: valores vivenciais (vivenciar algo ou alguém), valores criativos (criar obras artísticas ou científicas) e valores atitudinais (tomar uma postura perante uma situação que não se pode modificar, ou seja, transformar uma tragédia em uma realização humana). Nessa perspectiva, diverge do existencialismo 
Thiago Antonio Avellar de Aquino, Valdiney Gouveia Veloso, Andrei Alves de Aguiar, Tiago Deividy Bento Serafim, Alisson de Meneses Pontes, Gylmara de Araújo Pereira \& Ana Sandra Fernandes

pessimista (Camus, 2006; Sartre, 1997), que apregoa que a principal tarefa do ser humano seria suportar a falta de sentido da existência. Em contraposição, entende-se que o dever primordial do indivíduo seria o de encontrar um sentido para a sua vida (Frankl, 2008).

Sobre esses três pressupostos, Frankl (1989) desenvolve sua base teórica pautando-se em concepções filosóficas e clínicas. Outros autores também tentaram corroborar o conceito de realização de sentido e vazio existencial por meio de instrumentos mais objetivos. Entretanto, antes de comentar acerca desses instrumentos, faz-se necessário descrever o significado de vazio existencial enquanto construto.

\section{O construto vazio existencial}

De forma geral, denomina-se de vácuo existencial a sensação de vazio decorrente da percepção de que a vida não tem sentido, sendo a existência vivenciada como algo que não tem qualquer propósito ou valor (Frankl, 2008, 2011). Também pode ser compreendido como um tipo de niilismo privado, pois se constitui por uma postura de negação de quaisquer sentidos (Frankl, 2008). No que se refere à sua etiologia, Frankl (2011) atribui a duas perdas: dos instintos e das tradições. Destarte, o ser humano moderno sofreria por não mais saber o que quer e o que deve fazer de sua existência. Por conseguinte, faz o que os outros fazem (conformismo) ou o que os outros querem (totalitarismo). Estimase que $20 \%$ dos pacientes neuróticos sofrem de uma sensação de falta de sentido (Lukas, 1988). De forma geral, o vácuo existencial está associado com a perda da perspectiva de futuro, restringindo a percepção de um "para quê viver". Em decorrência, o indivíduo pode assumir uma forma de existência provisória, desencadeando um excesso de busca de prazer e poder; outras formas de sua manifestação são o tédio (não se interessar por algo) e a apatia (não tomar iniciativa para algo) (Frankl, 2011).

Outros sintomas também são ressaltados por Frankl (2005), sendo denominados de tríade da neurose de massa: depressão, agressão e toxicodependência. Frankl (1989) ainda reconhece que há formas dissimuladas de expressão do vazio existencial, como: (1) refugiar-se no trabalho para compensar a falta de realização pessoal; (2) tentar fugir de si mesmo, da vida simples e verdadeira, por meio do trabalho excessivo para não se deparar com o próprio deserto interior; e (3) o que se denomina como neurose dominical, em que o homem se sente incomodado ao chegar o domingo ou o fim de semana em que fica em casa e tem de conviver com a família, consigo mesmo e com o próprio vazio da existência. Isso é possível perceber, por exemplo, quanto tem lugar o decaimento psicofísico do aposentado, em razão de ele perder o sentido que encontrava no trabalho.

Por fim, Frankl (1989) assevera que o vácuo existencial não se constitui por si só em uma neurose, mas seria potencialmente patogênico. Para constituir-se como uma neurose torna-se necessário mais um elemento: o conflito. Nessa perspectiva, Frankl (2011) denomina tal neurose de noogênica, descrevendo-a como sendo "causada por um conflito em nível espiritual - um conflito ético ou moral [...]. A etiologia noogênica é formada pelo vácuo existencial, pela frustração existencial ou pela frustração da vontade de sentido" (p. 112).

\section{Medidas para avaliar a vontade de sentido}

Com o fim de validar o construto de vontade de sentido, tornou-se necessária a construção de instrumentos de medida que permitissem aferir o grau de realização e frustração existencial. Dessa forma, fez-se fundamental analisar algumas das suas principais medidas, sobretudo no que se refere aos seus aspectos psicométricos (evidências de validade e precisão). Descrevem-se, a seguir, três dos instrumentos que, presumivelmente, são os mais utilizados na literatura:

Teste Propósito de Vida (Pil-Teste)

O Teste de Propósito de Vida (Pil-Teste) foi proposto por Crumbaugh e Maholick (1964) com a finalidade de validar os pressupostos teóricos da Logoterapia e análise existencial. Harlow, Newcomb e Bentler (1987), por sua vez, elaboraram a versão Pil-R, uma versão revisada que contempla três fatores: desespero existencial [alfa de Cronbach $(\alpha)=0,69$ ], realização existencial $(\alpha=0,71)$ e vazio existencial $(\alpha=0,72)$. De forma geral, os 
Thiago Antonio Avellar de Aquino, Valdiney Gouveia Veloso, Andrei Alves de Aguiar, Tiago Deividy Bento Serafim, Alisson de Meneses Pontes, Gylmara de Araújo Pereira \& Ana Sandra Fernandes

itens dessa medida contemplam os seguintes aspectos: propósito na vida, satisfação com a própria vida, liberdade, medo da morte, ideias suicidas e se a vida vale a pena.

Desde que o Pil-Teste foi criado, vários foram os trabalhos desenvolvidos que se utilizaram dessa escala. Como exemplo, tem-se um estudo desenvolvido por Aquino et al. (2009) que verificou as relações entre o sentido da vida e a atitude religiosa, utilizando a versão proposta por Harlow et al. (1987). A amostra foi constituída por 300 participantes de ambos os sexos, apresentando três fatores: I = Vazio existencial $(\alpha=0,78), \| I=$ Realização existencial $(\alpha=0,75)$ e III $=$ Desespero existencial $(\alpha=0,65)$. Quando tais autores consideraram esse instrumento como unifatorial, seu alfa de Cronbach foi de 0,83. Vale salientar que nesse estudo propôs-se uma versão reduzida desse instrumento, constituída por 12 itens.

Outro estudo foi levado a cabo por Aquino et al. (2010), os quais objetivaram identificar as relações entre visões de morte com o nível de ansiedade perante a morte e o vazio existencial. Esse instrumento apresentou um fator geral formado por 14 itens, denominado de "vazio existencial", com cargas fatoriais superiores a $|0,30|$ e alfa de Cronbach 0,81. Nessa mesma direção, o estudo de Shek (1988), em contexto chinês, contando com 2.140 jovens estudantes do ensino médio, após uma análise fatorial exploratória sugeriu uma estrutura unifatorial cujo fator geral apresentou índice satisfatório de consistência interna (alfa de Cronbach $=0,90$ ).

Na esfera de saúde/doença, Marsh, Smith, Piek e Saunders (2003) averiguaram a estrutura do Pil-Teste por meio de análise fatorial confirmatória. O estudo contou com 357 australianos que bebiam socialmente e outras 137 pessoas que estavam em tratamento alcoólico. Para o primeiro grupo, os índices de ajustes foram considerados adequados, levando-se em conta os 20 itens $\left[\chi^{2}(115,36)=212,92\right.$, RMSEA $=0,049, \mathrm{GFI}=0,96$ e $A G F I=0,95]$. Para o segundo grupo, aquele que estava em tratamento, os índices de ajuste, de forma geral, mostraram-se aceitáveis $\left[\chi^{2}(108,14)\right.$ $=182,94$, RMSEA $=0,071, \mathrm{GFI}=0,93$ e $A G F I=0,90]$. Esses autores relataram a precisão desse instrumento apenas para o segundo grupo, que se mostrou satisfatória $(\alpha=0,95)$.

O estudo de Halama (2009), considerando uma amostra de 168 estudantes universitários eslovacos, identificou três fatores: excitação na vida $(\alpha=0,75)$, satisfação com a vida $(\alpha=0,74)$ e propósitos e objetivos $(\alpha=0,71)$. No contexto suíço, Jonsén et al. (2010) procuraram conhecer os parâmetros psicométricos do Pil-Teste junto uma amostra de 449 participantes. No caso, foi realizada uma análise fatorial exploratória que permitiu extrair três dimensões: sentido na existência, liberdade para criar significado na vida diária e vontade de encontrar significado em desafios futuros. A consistência interna foi aferida por meio do alfa de Cronbach, variando de 0,54 a 0,83 . Também foi verificada a possibilidade de esse instrumento ser considerado unifatorial, tendo o fator geral apresentado alfa de 0,83.

Ortiz, Cano e Trujillo (2012) administraram o Pil-Teste a 798 colombianos com a finalidade de testar a melhor estrutura fatorial (unifatorial, bifatorial ou trifatorial). O modelo com três fatores se apresentou como o mais adequado dos três, sugerindo as seguintes dimensões: metas na vida ( $\alpha=0,89)$, satisfação com a vida $(\alpha=0,87)$ e sensação de realização $(\alpha=0,69)$. Esses autores consideram a adequabilidade de tal estrutura tanto em função dos aspectos teóricos quanto clínicos.

De forma geral, esses estudos apresentam a possibilidade de considerar o sentido de vida como um construto multidimensional, embora autores como Aquino et al. (2009) e Chamberlain e Zika (1988) apontem também como plausível sua estrutura unifatorial. Portanto, observa-se que o número de fatores, bem como a sua análise semântica, embora coerente com os conceitos da análise existencial de Frankl (1989), apresentam-se de forma inconsistente, variando de acordo com a especificidade da amostra. Portanto, demanda-se analisar outras medidas, o que se leva a cabo a seguir.

Logo-Teste

Esse é outro instrumento que também teve o objetivo de validar o construto proposto por Frankl (1989), tendo sido apresentado por 
Thiago Antonio Avellar de Aquino, Valdiney Gouveia Veloso, Andrei Alves de Aguiar, Tiago Deividy Bento Serafim, Alisson de Meneses Pontes, Gylmara de Araújo Pereira \& Ana Sandra Fernandes

Elisabeth Lukas. Foi desenvolvida em contexto vienense (Áustria), no Instituto de Psicologia Experimental e Aplicada da Universidade de Viena (Lukas, 1988), constituindo-se em uma alternativa psicométrica para a aferição da vontade de sentido. Compreende um conjunto de 18 itens, distribuídos em três partes: (1) autoconhecimento noético (constituído por nove itens referentes a possibilidades de descoberta de sentido; e.g., em meu trabalho profissional, que realizo na atualidade ou para o qual estou me preparando, encontro a minha realização); (2) manifestação da frustração existencial (formado por sete itens; e.g., sentimento de raiva por que penso que tudo que realizei até agora foi inútil); e (3) autoavaliação a respeito de metas e objetivos, o que se alcançou e sua postura perante os mesmos. Com o propósito de validar esse instrumento, sua autora o administrou em amostra constituída por 340 pessoas, das quais 55 pacientes de uma clínica psiquiátrica em Viena; sua precisão foi avaliada por meio do método Sperman-Brown, resultando em um índice de 0,84 (Lukas, 1988).

No contexto brasileiro, um estudo realizado por Andrade (2001) avaliou a dimensão valorativa do sentido da vida, objetivando conhecer a relação entre os valores humanos e o sentido da vida. Nesse caso, foi administrado o Logo-Teste em um grupo de 400 pessoas da população geral, distribuídas igualmente em três áreas geográficas da cidade de João Pessoa, segundo o nível socioeconômico (baixo, médio e alto). Como resultados preliminares, essa autora observou uma estrutura unifatorial do conjunto de 11 itens, explicando 12,2\% da variância total; sua precisão foi aferida por meio do alfa de Cronbach, que apresentou índice de 0,46, considerado abaixo do que tem sido recomendado na literatura $(0,70$; Pasquali, 2003).

Noblejas de la Flor (1999), tomando em conta uma amostra de 841 pessoas que apresentaram idade entre 16 e 60 anos, na Espanha, verificou tanto a confiabilidade do Pil-Teste quanto do Logo-Teste. Segundo essa autora, o Pil-Teste apresentou uma confiabilidade alta, com alfa de 0,88; no entanto, reforçando estudo previamente citado, o índice de consistência interna apresentado para o Logo-Teste foi baixo (alfa de Cronbach de 0,51). Portanto, como instrumento diagnóstico, esse apresenta uma confiabilidade inadequada para aferir o construto em questão. Nesse sentido, torna-se premente o surgimento de novas medidas que permitam conhecer melhor essa categoria nosológica.

\section{Questionário Sentido de Vida (QSV)}

Segundo Steger (2009), as pesquisas acerca da percepção do sentido da vida ora enfatizam a crença da presença ou ausência de um propósito para a existência, ora dão ênfase à compreensão de como e onde as pessoas encontram esse sentido, indicando o grau de engajamento nessa busca. Em uma revisão da literatura, esse autor identificou dois métodos de pesquisa sobre esse mesmo construto. O primeiro reúne uma série de respostas à seguinte pergunta: "O que lhe traz sentido à vida?" (Ebersole \& Devogler, 1981), permitindo identificar várias fontes de sentido da vida, tais como: relacionamentos, crenças religiosas, saúde e crescimento pessoal; já o segundo método consiste em inventário contendo uma lista descrevendo fontes de sentido na vida em potencial e os entrevistados classificam o seu grau de importância (Bar-Tur, Savaya \& Prager, 2001).

Tendo em vista que poucas pesquisas levam em conta a busca das pessoas pelo sentido, Steger et al. (2006) desenvolveram uma nova medida de sentido da vida, formada por dez itens, na qual discriminam duas dimensões: a busca e a presença de sentido. A busca do sentido se refere àquelas pessoas que estão tentando compreender ou aumentar o propósito de sua vida, enquanto que a presença diz respeito àquelas que estão engajadas em um sentido ao longo da vida. Esses autores definem o sentido na vida como aquilo que faz sentir que a vida possui um sentido na existência, levando em conta o seu próprio critério de significado.

De acordo com a estrutura previamente descrita, Steger et al. (2006) testaram em três amostras estadunidenses, por meio de uma análise fatorial confirmatória, um modelo bifatorial ortogonal. Os resultados dessas análises indicaram um ajuste aceitável do modelo proposto: amostra $1\left[\chi^{2}(\mathrm{n}=153)=57,68\right.$, $G F I=0,93, A G F I=0,89, C F I=0,97 \mathrm{e}$ RMSEA $=0,07]$, amostra $2\left[\chi^{2}(\mathrm{n}=279)=\right.$ $56,04, \mathrm{GFI}=0,97, \mathrm{AGFI}=0,96, \mathrm{CFI}=0,99$ 
Thiago Antonio Avellar de Aquino, Valdiney Gouveia Veloso, Andrei Alves de Aguiar, Tiago Deividy Bento Serafim, Alisson de Meneses Pontes, Gylmara de Araújo Pereira \& Ana Sandra Fernandes

e $R M S E A=0,04]$ e amostra $3\left[\chi^{2}(\mathrm{n}=402)=\right.$ $149,59, \mathrm{GFI}=0,93, \mathrm{AGFI}=0,89, \mathrm{CFI}=0,93 \mathrm{e}$ RMSEA $=0,09]$, apesar de nessa última amostra não serem excelentes (Hu \& Bentler, 1999; Kline, 2010; Tabachnick \& Fidell, 1996), tais indicadores de ajuste são ao menos meritórios, consoantes com os valores observados nas outras duas amostras. Nos três estudos, os alfas de Cronbach para os dois fatores foram sempre superiores a 0,80. Nesse sentido, os autores supracitados consideraram que o instrumento ora descrito reuniu evidências psicométricas favoráveis, contribuindo com pesquisas acerca do bem-estar, sendo útil no contexto do aconselhamento psicológico.

Considerando os instrumentos descritos previamente, o Questionário Sentido de Vida se mostrou o mais parcimonioso, contando com menor número de itens, porém favorecendo pensar no construto como tendo mais de uma dimensão, apreendendo-se o conceito de sentido de vida como busca e presença. Além disso, ao menos no contexto estadunidense, dito instrumento reuniu parâmetros psicométricos satisfatórios quanto a evidências de validade fatorial e consistência interna. Contudo, em seu estudo, Steger et al. (2006) unicamente testaram o modelo bifatorial, sem contrastá-lo com um modelo aninhado alternativo, isto é, unifatorial. Dessa forma, o objetivo do presente estudo foi adaptar esse questionário para o contexto brasileiro, reunindo evidências de sua validade fatorial, validade convergente e consistência interna, comparando os modelos uni e bifatorial. Dois estudos foram realizados com tais propósitos.

\section{Estudo 1. Evidências de validade fatorial e precisão}

Nesse estudo inicial o propósito foi realizar análises de natureza confirmatória, averiguando-se a estrutura fatorial do Questionário Sentido de Vida (QSV). Concretamente, comparam-se os modelos uni e bifatorial.

\section{Método}

\section{Participantes}

Participaram desse estudo 414 estudantes universitários de João Pessoa, PB, com idade média de 28,2 anos $(d p=9,50$; amplitude de 18 a 63 anos), sendo a maioria do sexo feminino $(63,5 \%)$. No que se refere ao estado civil, 30\% se declararam casados, 52,2\% solteiros, $10 \%$ separados e $6,8 \%$ outros; os que não responderam essa pergunta totalizaram 1\%.

\section{Instrumento}

Os participantes responderam um instrumento com duas partes: (1) Questionário Sentido de Vida (QSV), formado por dez itens avaliados em escala de 7 pontos, variando de $\mathbf{1}=$ Totalmente falso a 7 = Totalmente verdadeiro. De acordo com Steger et al. (2006), esse instrumento apresenta dois fatores: (1) presença de sentido (e.g., Eu compreendo o sentido da minha vida) e busca de sentido (e.g., Eu estou procurando alguma coisa que faça com que minha vida tenha sentido); e (2) informações demográficas (estado civil, sexo e idade).

Inicialmente, solicitou-se ao professor Michael F. Sterger, autor do QSV, a autorização para adaptá-lo ao contexto brasileiro. Recebida resposta favorável, procedeu-se à sua tradução do inglês para o português, contando com a colaboração de duas pessoas bilíngues, sendo uma estadunidense e outra brasileira. Posteriormente solicitou-se a uma terceira pessoa, especialista em língua inglesa, que comparasse as versões traduzidas para o português com o original em inglês. Adicionalmente, efetuou-se uma tradução reversa (back-translation) por uma quarta pessoa, igualmente bilíngue. Constatou-se, então, que a versão em português apresentava equivalência semântica com o original em inglês. Finalmente, checaram-se evidências de sua validade semântica em amostra de dez estudantes universitários, oportunidade em que se aferiu a compreensão de instruções, escala de resposta e conteúdo dos itens.

\section{Procedimento}

Os instrumentos foram respondidos em contexto coletivo de sala de aula, porém individualmente. Entretanto, esse processo unicamente teve lugar após o consentimento dos professores e, no caso da aplicação em si, depois de dadas as explicações quanto ao objetivo do estudo e de ter-se informado aos participantes sobre como deveriam proceder para o preenchimento. A pesquisa seguiu todas as recomendações éticas de pesquisas 
Thiago Antonio Avellar de Aquino, Valdiney Gouveia Veloso, Andrei Alves de Aguiar, Tiago Deividy Bento Serafim, Alisson de Meneses Pontes, Gylmara de Araújo Pereira \& Ana Sandra Fernandes

com seres humanos, tendo os participantes assinado Termo de Consentimento Livre e Esclarecido. Em média, os respondentes levaram entre 5 e 10 minutos para concluir sua participação na pesquisa.

Análise de dados

Dois pacotes estatísticos foram utilizados: SPSS (versão 16) e AMOS (versão 7). Utilizou-se o primeiro para calcular estatísticas descritivas (medidas de tendência central e dispersão) e consistência interna (alfa de Cronbach). No caso, o segundo programa foi empregado para realizar as análises fatoriais confirmatórias, comparando os modelos uni e bifatorial. Adotou-se a matriz de covariância como entrada, assumindo o estimador ML (Maximum Likelihood). Empregaram-se os seguintes estimadores para avaliar a adequação dos modelos (Byrne, 2010; Garson, 2003; Hu \& Bentler, 1999; Kelloway, 1998; Kline, 2010; Tabachnick \& Fidell, 1996; Van de Vijver \& Leung, 1997):

$\mathrm{O} \chi^{2}$ (qui-quadrado) testa a probabilidade de o modelo teórico se ajustar aos dados; valores altos são indicativos de um não ajustamento. Entretanto, o índice mais utilizado é a razão entre o qui-quadrado e o seu grau de liberdade $\left(\chi^{2} / \mathrm{gl}\right)$. Valores entre 2 e 3 sugerem um ajustamento adequado, admitindo-se até 5 .

O Goodness of Fit Index (GFI) e o Adjusted Goodness of Fit Index (AGFI) sugerem a proporção de variância-covariância nos dados explicada pelo modelo; valores iguais ou superiores a 0,90 indicam um ajuste adequado. O mesmo critério de aceitabilidade vale para o Comparative Fit Index (CFI), que compara a matriz de covariância predita pelo modelo com aquela observada.
A Root Mean Square Error of Approximation (RMSEA), juntamente com seu respectivo intervalo de confiança de 90\% (IC90\%), permite tomar decisão a respeito do modelo testado; consideram-se aceitáveis valores que se encontrem no intervalo de 0,05 a 0,08, podendo ser tolerado até 0,10.

Finalmente, são considerados três indicadores para comparar os modelos. A diferença entre os qui-quadrados dos modelos e seus respectivos graus de liberdade $\left(\Delta \chi^{2}\right)$ é um teste estatístico que, diferente dos indicadores GFI e CFI, por exemplo, apresenta nível de significância, admitindo-se $p<0,05$, penalizando aquele com maior valor de $\chi^{2}$. Além desse, tem também o Expected Cross-Validation Index (ECVI) e o Consistent Akaike Information Criterion (CAIC), favorecendo decidir o modelo mais adequado; os melhores modelos apresentam valores menores desses dois indicadores de ajuste.

\section{Resultados}

Inicialmente, verificou-se a pertinência de realizar uma análise fatorial, observando-se indicadores que apoiam esse tipo de análise $[K M O=0,89$; Teste de Esfericidade de Bartlett, $\left.\chi^{2}(45)=2.178,35, p<0,001\right]$. Tomando-se em conta a versão original do Questionário Sentido de Vida e a teoria que o fundamenta, decidiu-se realizar uma análise fatorial com a definição dos itens por fator congruente com a proposta de Steger et al. (2006). Porém, além do modelo bifatorial (presença e busca de sentido), testou-se ainda o modelo unifatorial, com todos os itens saturando em um mesmo fator geral (sentido de vida). Os resultados dessas análises podem ser observados na Tabela 1.

Tabela 1. Comparação de modelos fatoriais do Questionário Sentido de Vida

\begin{tabular}{ccccccccccc}
\hline Modelos & $\chi^{2}$ & gl & $\chi^{2} / \mathbf{g l}$ & GFI & AGFI & CFI & $\begin{array}{c}\text { RMSEA } \\
(\text { IC90\%) }\end{array}$ & CAIC & ECVI & $\Delta \chi^{2}$ (g.l) \\
\hline Unifatorial & 790,39 & 35 & 22,6 & 0,63 & 0,42 & 0,65 & $\begin{array}{c}0,22 \\
(0,22-0,24)\end{array}$ & 930,91 & 2,01 & ------- \\
Bifatorial & 137,5 & 34 & 4,0 & 0,94 & 0,90 & 0,95 & $\begin{array}{c}0,086 \\
(0,07-0,10)\end{array}$ & 285,04 & 0,44 & $652,89^{*}$ \\
& & & & & & & & $(1)$ \\
\hline
\end{tabular}

Nota: $\chi^{2} / g$ l - razão qui-quadrado/graus de liberdade; GFI - Goodness of Fit Index; AGFI - Adjusted Goodness of Fit Index; CFI - Comparative Fit Index; RMSEA - Root Mean Square Error of Approximation; IC90\% = Intervalo de Confiança de 90\%; CAIC = Consistent Akaike Information Criterion; ECVI = Expected Cross-Validation Index. ${ }^{*} \mathrm{p}<0,001$. 
Thiago Antonio Avellar de Aquino, Valdiney Gouveia Veloso, Andrei Alves de Aguiar, Tiago Deividy Bento Serafim, Alisson de Meneses Pontes, Gylmara de Araújo Pereira \& Ana Sandra Fernandes

De acordo com essa tabela, comparando-se os indicadores de ajuste do modelo unifatorial $\left[\chi^{2}(35)=790,39, p<0,001, \chi^{2} / g l=22,58\right.$, $\mathrm{GFI}=0,63, A G F I=0,42, C F I=0,65$, RMSEA $=0,22]$ com os do modelo bifatorial $\left[\chi^{2}(34)=137,50, p<0,001, \chi^{2} / g l=4,04\right.$, $\mathrm{GFI}=0,94, A G F I=0,90, C F I=0,95 \mathrm{e}$ RMSEA $=0,086]$, percebe-se que esse último é mais promissor. De fato, o $\Delta \chi^{2}$ foi significativo $(p<0,001)$, corroborando o modelo bifatorial, que apresentou menor qui-quadrado. Reforçando esse achado, os valores de ECVI e CAIC para esse modelo foram claramente inferiores aos observado para o unifatorial. Pode-se visualizar a solução bifatorial na Figura 1 a seguir.

De forma geral, as saturações variaram de $-0,53$ a 0,85 , todas estatisticamente diferentes de zero $(\lambda \neq 0 ; z>1,96, p<0,05)$. Para o primeiro fator, presença de sentido, fixou-se em 1 a saturação do item 1 (Eu compreendo o sentido da minha vida), observando-se saturações variando de -0,53 (item 9: Minha vida não tem um propósito claro) a 0,85 (item 4: Minha vida tem um sentido claro), apresentando alfa de Cronbach $(\alpha)$ de 0,85 . No caso do segundo fator, busca de sentido, fixou-se em 1 a saturação do item 2 (Eu estou procurando alguma coisa que faça com que minha vida tenha sentido), tendo sido observadas saturações variando de 0,74 (item 7: Eu estou sempre procurando por algo que faça com que minha vida seja significante) a 0,83 (item 10: Eu estou procurando um sentido em minha vida), observando-se $\alpha=0,89$.

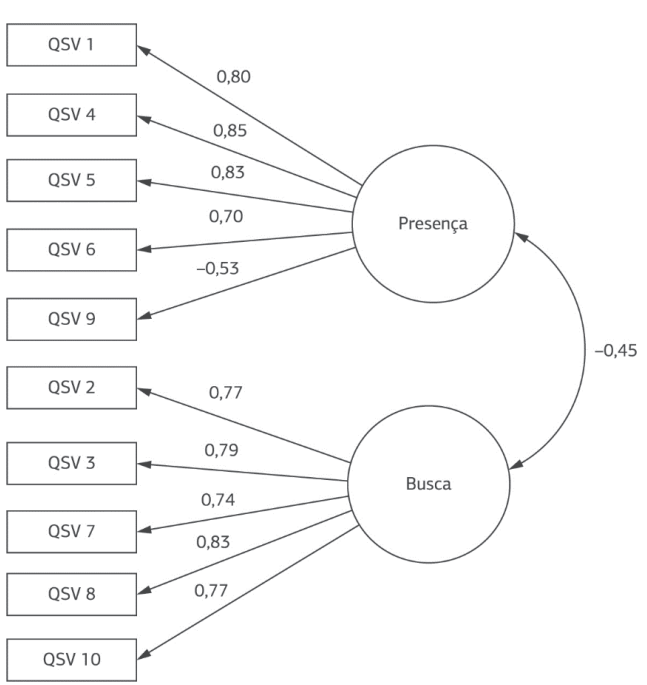

Figura 1. Estrutura bifatorial do Questionário Sentido de Vida (QSV).

\section{Discussão parcial}

Conforme se observa nos resultados, coerente com a proposta de Steger et al. (2006), o Questionário Sentido de Vida apresenta dois fatores, nomeados como presença de sentido e busca de sentido. Os indicadores de ajuste da análise fatorial confirmatória atenderam ao estabelecido na literatura (Byrne, 2010; Garson, 2003), sendo a solução bifatorial a única adequada; a unifatorial se mostrou pouco plausível. Além disso, os coeficientes de consistência interna dos dois fatores foram acima do ponto de corte recomendado na literatura (Pasquali, 2003), sendo inclusive equivalentes aos observados por seus autores. Portanto, existem evidências de validade fatorial e precisão dessa medida. Entretanto, resta conhecer em que medida pode-se correlacionar com construtos correlatos (validade convergente), o que motivou o segundo estudo.

\section{Estudo 2. Evidências de validade convergente}

Esse estudo buscou, primordialmente, verificar em que medida o Questionário Sentido de Vida reúne evidências de validade convergente, permitindo cobrir aspectos essenciais do construto sentido de vida, mas sem se solapar com outros instrumentos que também o meçam, justificando seu uso.

\section{Método}

\section{Participantes}

Participaram desse estudo 201 estudantes universitários de duas instituições, uma pública e outra privada, de João Pessoa, PB, sendo a maioria constituída por pessoas do sexo feminino $(74,6 \%)$ e casada $(61,2 \%)$. Esses indicaram ter idade entre 18 e 63 anos, apresentando média de 26,7 anos $(d p=9,56)$.

\section{Instrumentos}

Os participantes receberam um livreto para responder, formado por três medidas: (1) Questionário Sentido de Vida, descrito previamente; (2) Pil-Teste (Purpose in Life), versão revisada de Harlow et al. (1987), 
Thiago Antonio Avellar de Aquino, Valdiney Gouveia Veloso, Andrei Alves de Aguiar, Tiago Deividy Bento Serafim, Alisson de Meneses Pontes, Gylmara de Araújo Pereira \& Ana Sandra Fernandes

correspondendo ao PIL-R; a versão utilizada foi a proposta por Aquino et al. (2009), composta por 12 itens, assumida como unifatorial $(\alpha=0,83)$. No caso, pontuações maiores indicam vazio existencial e menores sinalizam maior presença de realização existencial; e (3) Escala de Percepção Ontológica do Tempo, elaborada por Aquino (2009) a partir do modelo teórico de Frankl (1989) no que se refere à temporalidade ontológica como marcadora do sentido de vida. Compõe-se de dez itens, sendo três para cada uma das perspectivas temporais: passado (e.g., Sinto-me realizado com o que alcancei; $\alpha=0,66)$, presente (e.g., Vejo sempre um motivo para estar no mundo; $\alpha=0,76$ ) e futuro (e.g., Vejo muitas possibilidades de escolha; $\alpha=0,75$ ), e um último que visava identificar a (in)satisfação geral com o eu ao longo do tempo (Tenho que admitir que há uma grande distância entre quem eu sou e quem eu poderia ser). Os participantes deviam respondê-los em escala de cinco pontos, variando de $\mathbf{1}=$ Discordo totalmente a $\mathbf{5}$ = Concordo totalmente. No final, os participantes responderam três perguntas demográficas (estado civil, sexo e idade).

Procedimento e análise dos dados

A coleta de dados ocorreu de forma semelhante à primeira pesquisa, respeitando-se os princípios éticos e assegurando-se a assinatura do Termo de Consentimento Livre e Esclarecido por parte dos participantes. Em média, foram necessários de 15 a 20 minutos para responder os instrumentos. Calcularam-se estatísticas descritivas e correlações de Pearson.

\section{Resultados}

Os resultados das correlações entre as escalas podem ser visualizados na Tabela 2. Constata-se que o vazio existencial se correlacionou negativamente com presença de sentido $(r=-0,71, p<0,001)$ e positivamente com busca de sentido ( $r=0,27, p<0,05)$. Portanto, quanto maior a pontuação em presença de sentido menor o vazio existencial.

As três dimensões da temporalidade se correlacionaram negativamente com o vazio existencial, como se indica: passado $(r=-0,61, p<0,001)$, presente $(r=-0,68$, $p<0,001)$ e futuro $(r=-0,59, p<0,001)$. Desse modo fica evidente que quanto mais positiva for a percepção da temporalidade, menor será o vazio existencial experimentado pela pessoa. Contrariamente, é preciso destacar que a dimensão presença de sentido se correlacionou positivamente com as três dimensões da temporalidade: passado $(r=0,47$, $p<0,001)$, presente $(r=0,55, p<0,001)$ e futuro $(r=0,48, p<0,001)$. Finalmente, a dimensão do QSV denominada como busca de sentido se correlacionou negativamente com as dimensões de temporalidade passado $(r=-0,17, p<0,01)$ e presente $(r=-0,14$, $p<0,05)$. Nesse sentido, entende-se que quanto maior a pontuação em presença de sentido mais positiva é a percepção do tempo; inversamente, quanto maior a pontuação em busca de sentido, mais negativa é a percepção de passado e presente.

\section{Discussão}

O objetivo deste artigo foi conhecer evidências psicométricas do Questionário Sentido de Vida (QSV) no contexto brasileiro. Foram averiguadas, sobretudo, evidências de suas validades fatorial e convergente, assim como seu índice de consistência interna. A título de organização, os resultados são discutidos de acordo com cada um desses parâmetros.

\section{Validade fatorial}

Procurou-se verificar a estrutura fatorial do Questionário Sentido de Vida em português tomando em conta uma amostra de estudantes

Tabela 2. Correlações entre os fatores do QSV, Pil-Teste e EPOT

\begin{tabular}{lllllc}
\hline & Passado & Presente & Futuro & $\begin{array}{c}\text { Presença } \\
\text { de sentido }\end{array}$ & $\begin{array}{c}\text { Busca de } \\
\text { sentido }\end{array}$ \\
\hline Presença de sentido & $0,47^{* * *}$ & $0,55^{* * *}$ & $0,48^{* * *}$ & - & - \\
Busca de sentido & $-0,17^{* *}$ & $-0,14^{*}$ & $-0,05$ & $-0,29^{* * *}$ & - \\
Vazio existencial & $-0,61^{* * *}$ & $-0,68^{* * *}$ & $-0,59^{* * *}$ & $-0,71^{* *}$ & $0,27^{* * *}$ \\
\hline
\end{tabular}

${ }^{*} \mathrm{p}<0,05 .{ }^{* *} \mathrm{p}<0,01 .{ }^{* * *} \mathrm{p}<0,001$. 
Thiago Antonio Avellar de Aquino, Valdiney Gouveia Veloso, Andrei Alves de Aguiar, Tiago Deividy Bento Serafim, Alisson de Meneses Pontes, Gylmara de Araújo Pereira \& Ana Sandra Fernandes

universitários. Dois modelos foram testados, segundo a perspectiva de Steger et al. (2006) sobre a busca e a presença de sentido. O modelo que sugere uma estrutura bifatorial se ajustou satisfatoriamente em comparação com o modelo unifatorial, atendendo a critérios empíricos e teóricos (Byrne, 2010; Kline, 2010). De forma específica, com relação aos índices de ajustes (GFI, CFI e AGFI), o modelo bifatorial apresentou-se mais promissor, considerando que todos foram próximos de 1 (0,90 e 0,95), sendo que seu RMSEA se situou em intervalo de confiança aceitável. Com relação ao $\chi^{2} / g$ l, obteve-se valor igualmente dentro do recomendado (Marsh \& Hocevar, 1995). Por fim, os índices CAIC e EVIC, do mesmo modo que o $\Delta \chi^{2}$, apoiaram o modelo bifatorial, que apresentou valores mais adequados do que aqueles correspondentes ao unifatorial (Byrne, 2010). Portanto, corroborou-se o modelo proposto por Steger et al. (2006), sugerindo-se que o sentido de vida é melhor representado pelos fatores busca de sentido e presença de sentido.

\section{Validade convergente}

Inicialmente, partiu-se da premissa de que o QSV e o Pil-Teste se propõem a medir o mesmo construto (realização e vazio existencial). Destarte, uma associação entre essas duas medidas seria uma evidência de validade convergente do QSV; coerentemente, foi precisamente isso que se observou, com essas medidas compartilhando cerca de 50\% da variância. Porém, esperava-se uma correlação "moderada" entre vazio existencial e busca de sentido ( $r=0,27, p<0,001)$, que também foi constatada; esclarece-se que esse coeficiente, embora possa ser pensado como pequeno, considerando-se padrões estatísticos gerais (Hair, Anderson, Tatham \& Black, 2005), compreende o valor médio comumente observado em estudos com construtos psicológicos (Gouveia, Santos \& Milfont, 2009). Tais achados corroboram aqueles de Steger et al. (2006), que encontraram também associações entre o fator busca de sentido do QSV e o índice geral do Pil-Teste.

Outra medida utilizada foi a Escala de Percepção Ontológica do Tempo (EPOT), sugerindo que uma percepção positiva do passado, presente e futuro estaria associada com o fator presença do sentido, o que seria consistente com a perspectiva teórica de Frankl (1989). Esse, em sua análise existencial, compreende que a sensação de um sentido para a vida pode ser advinda de valores realizados no passado ou no presente, assim como a percepção de novas possibilidades de sentidos que estariam no campo perceptivo do futuro do indivíduo. Congruentemente, estudos apontam relações positivas da presença de sentido com a satisfação com a vida, porém inversa com presença de sentido e depressão (Steger et al., 2006).

No que tange à busca de sentido, essa medida se correlacionou apenas com o passado e o presente. Embora as correlações tenham sido baixas, são também importantes. Desse modo, os resultados sugerem que a busca de sentido decorre de uma insatisfação existencial com o presente e o passado. Isso é consistente com o que pensa Fizzotti (1998), segundo o qual o ser humano não se realiza apenas pelo fato de estar no mundo, mas deseja encontrar e realizar sentidos. $\mathrm{O}$ fato de não encontrar significados no passado ou no presente o levaria a uma busca de sentido e isso reproduz a concepção de Frankl (1978) acerca da vontade de sentido como motivação primária. De forma geral, esses achados apresentam evidências de validade convergente do QSV.

Consistência interna

Os índices de consistência interna do QSV podem ser considerados satisfatórios, pois foram acima do ponto de corte comumente recomendado na literatura, ou seja, $\alpha \geq 0,70$ (Nunnally, 1991; Pasquali, 2003). Indica-se, então, que esse instrumento poderá ser adequadamente empregado em pesquisa e, se assim desejar o leitor, em contexto clínico ou intervencionista, favorecendo práticas de orientação no marco da Logoterapia (Frankl, 1989).

Em resumo, esses achados apontam evidências de validade fatorial, convergente e consistência interna, recomendando a sua utilização e aplicabilidade no contexto brasileiro acerca de questões existenciais sobre o sentido da vida. Ademais, ressalta-se o caráter parcimonioso do QSV, sobretudo quando comparado com medidas clássicas (Pil-Teste e Logo-Teste). Nesse 
Thiago Antonio Avellar de Aquino, Valdiney Gouveia Veloso, Andrei Alves de Aguiar, Tiago Deividy Bento Serafim, Alisson de Meneses Pontes, Gylmara de Araújo Pereira \& Ana Sandra Fernandes

sentido, confia-se oferecer uma ferramenta útil para aplicação futura.

\section{Considerações finais}

Faz-se importante, nesse momento, pontuar as limitações potenciais dos estudos realizados. Ressalta-se, pois, o fato de as amostras terem sido de conveniência (não probabilísticas), considerando pessoas com nível de instrução elevado (estudantes universitários). Resta, no entanto, ponderar que se pretendia unicamente contar com evidências de adequação psicométrica do QSV, avaliando a possibilidade de identificar sua estrutura fatorial. Nesse sentido, entenda-se essa limitação como um desafio, estimulando estudos que visem adequá-lo para outros grupos.
Por fim, sugere-se realizar estudos futuros para ampliar evidências de validade convergente, utilizando-se outras medidas, como, por exemplo, a Escala Dimensional dos Recursos Noológicos (Ortiz, Buitrago \& Osma, 2010), a Escala Dimensional do Sentido de Vida (Ortiz, Trujillo, Castillo \& Osma, 2011) e/ou a Escala Existencial (Längle, Orgler \& Kundi, 2003). Quiç̧á valeria a pena criar um conjunto comum de itens para identificar dimensões congruentes nas diversas medidas, conhecendo-se o que são de fato atributos principais ou meramente fatores espúrios, pouco preponderantes, variando de um contexto a outro. Isso, provavelmente, contribuiria no sentido de aprimorar as medidas e os modelos de sentido da vida, representando avanços nessa área de pesquisa e prática. Será igualmente importante conhecer sua estabilidade temporal (testereteste), avaliando em que medida o construto sentido de vida é afetado pelo tempo. 


\section{Thiago Antonio Avellar de Aquino}

Doutor em Psicologia Social pela Universidade Federal da Paraíba, João Pessoa - PB. Brasil. Docente da Universidade Federal da Paraíba, João Pessoa - PB. Brasil.

E-mail: logosvitae@ig.com.br

\section{Valdiney Gouveia Veloso}

Doutor em Psicologia Social pela Universidad Complutense de Madrid, Madri, Espanha. Docente da Universidade Federal da Paraíba, João Pessoa - PB. Brasil.

E-mail:vvgouveia@gmail.com

\section{Andrei Alves de Aguiar}

Mestre em Ciência das Religiões pela Universidade Federal da Paraíba, João Pessoa - PB. Brasil. E-mail: andreipsicologia@hotmail.com

\section{Tiago Deividy Bento Serafim}

Mestre em Ciência das Religiões pela Universidade Federal da Paraíba, João Pessoa - PB.

Brasil. Docente da Faculdade Vale do Salgado, Fortaleza - CE. Brasil.

E-mail: tiagodeividy@gmail.com

\section{Alisson de Meneses Pontes}

Mestre em Ciência das Religiões pela Universidade Federal da Paraíba, João Pessoa - PB.

Brasil. Docente das Faculdades Integradas de Patos, João Pessoa - PB. Brasil.

E-mail: alisson.mpontes@hotmail.com

\section{Gylmara de Araújo Pereira}

Mestre em Ciência das Religiões pela Universidade Federal da Paraíba, João Pessoa - PB. Brasil. E-mail: gylmaradeapereira@gmail.com

\section{Ana Sandra Fernandes}

Mestre em Ciência das Religiões pela Universidade Federal da Paraíba, João Pessoa - PB. Brasil. E-mail: anasfernandes25@hotmail.com

\section{Endereço para envio de correspondência:}

Universidade Federal da Paraíba. Cidade Universitária. CEP 58051-900 - João Pessoa, PB - Brasil.

Recebido 27/08/2012, Aprovado 11/04/2013. 
Thiago Antonio Avellar de Aquino, Valdiney Gouveia Veloso, Andrei Alves de Aguiar, Tiago Deividy Bento Serafim, Alisson de Meneses Pontes, Gylmara de Araújo Pereira \& Ana Sandra Fernandes

Referências

Abbagnano, N. (1976). História da filosofia (Vol. 12). Lisboa: Editorial Presença.

Adler, A. (1998). What life should mean to you. Minnesota: Hazelden (Trabalho original publicado em 1931).

Andrade, M. W. C. L. (2001). A dimensão valorativa do sentido da vida. Dissertação de Mestrado (Psicologia Social), Departamento de Psicologia, Universidade Federal da Paraíba, João Pessoa, PB.

Aquino, T. A. A. (2009). Atitudes e intenções de cometer suicídio: seus correlatos existenciais e normativos. Tese de Doutorado (Psicologia Social), Departamento de Psicologia, Universidade Federal da Paraíba, João Pessoa, PB.

Aquino, T. A. A., Correia, A. P. M., Marques, A. L. C., Sousa, C. G., Freitas, H. C. A., Araújo, I. F. et al. (2009). Atitude religiosa e sentido da vida: Um estudo correlacional. Psicologia Ciência e Profissão, 29, 228-243.

Aquino, T. A. A., Serafim, T. D. B., Silva, H. D. M., Barbosa, E. L., Cirne, E. A., Ferreira, F. R., et al (2010). Visões de morte, ansiedade e sentido da vida: um estudo correlacional. Psicologia Argumento, 63, 289-302.

Bar-Tur, L., Savaya, R., \& Prager, E. (2001). Sources of meaning in life for young and old Israeli Jews and Arabs. Journal of Aging Studies, 15(3), 253-269. http://dx.doi.org/10.1016/ S0890-4065(01)00022-6.

Baumeister, R. (1991). Meanings of life. New York: The Guilford Press.

Boss, M. (1976). Análise Existencial. Daseinsanalyse: Como a Daseinsanalyse entrou na psiquiatria. Revista Daseinsanalyse, 2, 5-23.

Buber, M. (2001). Eu e tu (N. A. von Zuben, trad.). São Paulo: Centauro. (Trabalho original publicado em 1923).

Byrne, B. (2010). Structural equation modeling with Amos: basic concepts, applications and programming (2nd ed.). New York: Routledge, Taylor \& Francis.

Camus, A. (2006). O mito de Sísifo (3. ed., P. Whacht \&A. Roitman, trad.). Rio de Janeiro/São Paulo: Record. (Trabalho original publicado em 1942).

Chamberlain, K., \& Zika, S. (1988). Measuring meaning in life: examination of three scales. Personality and Individual Differences, 9(3), 589-596. http://dx.doi.org/10.1016/01918869(88)90157-2.
Crumbaugh, J. C., \& Maholick, L. T. (1964). Na experimental study in existentialism: the psychometric approach to Frankl's concept of noogenic neurosis. Journal of Clinical Psychology, 20(2), 200-207. http://dx.doi. org/10.1002/1097-4679(196404)20:2<200::AIDJCLP2270200203>3.0.CO;2-U. PMid:14138376

Ebersole, P., \& Devogler, K. L. (1981). Meaning in life: category self-ratings. The Journal of Psychology, 107(2), 289-293. http://dx.doi.or g/10.1080/00223980.1981.9915236.

Fizzotti, E. (1998). Os ritos de cura como autorealização e como busca de sentido. In F. Dal Pino (Orgs.), Liturgia e terapia: a sacramentalidade a serviço do homem na sua totalidade (pp. 235-275). São Paulo: Paulinas.

Frankl, V. E. (1978). Fundamentos antropológicos da psicoterapia (R. Bittencourt, trad.). Rio de Janeiro: Zahar Editores. (Trabalho original publicado em 1975).

Frankl, V. E. (1989). Psicoterapia e sentido da vida (A. M. Castro, trad.). São Paulo: Quadrante. (Trabalho original publicado em 1946).

Frankl, V. E. (1991). A psicoterapia na prática (C. M. Caon, trad.). Campinas: Papirus. (Trabalho original publicado em 1947).

Frankl, V. E. (2005). Um sentido para a vida: psicoterapia e humanismo (13. ed., V. H. Lapenta, trad.). São Paulo: Idéias e Letras. (Trabalho original publicado em 1978).

Frankl, V. E. (2008). Em busca de sentido: um psicólogo no campo de concentração (W. O. Schlupp \& C. C. Aveline, trad.). São Leopoldo: Sinodal. (Trabalho original publicado em 1946).

Frankl, V. E. (2011). A vontade de sentido: fundamentos e aplicações da logoterapia (I. S. Pereira, trad.). São Paulo: Paulus. (Trabalho original publicado em 1969).

Freud, S. (1976). Novas conferências introdutórias sobre Psicanálise. In S. Freud, Edição Standard Brasileira das Obras Psicológicas Completas de Sigmund Freud (Vol. XX, pp. 17-220). Rio de Janeiro: Imago (Trabalho original publicado em 1933).

Garson, G. D. (2003). PA 765 Statnotes: an online textbook. Recuperado em 15 de março de 2010, de http://www2.chass.ncsu.edu/garson/ pa765/statnote.htm.

Gouveia, V. V., Santos, W. S., \& Milfont, T. L. (2009). O uso da estatística na avaliação psicológica: 
Thiago Antonio Avellar de Aquino, Valdiney Gouveia Veloso, Andrei Alves de Aguiar, Tiago Deividy Bento Serafim, Alisson de Meneses Pontes, Gylmara de Araújo Pereira \& Ana Sandra Fernandes

comentários e considerações práticas. In C. S. Hutz. (Org.), Avanços e polêmicas em avaliação psicológica: em homenagem a Jurema Alcides Cunha (pp. 127-155). São Paulo: Casa do Psicólogo.

Hair, J. F. J., Anderson, R. E., Tatham, R. L., \& Black, W. C. (2005). Análise multivariada de dados. Porto Alegre: Bookman.

Halama, P. (2009). The Pil test in a Slovak sample: internal consistency and factor structure. International Forum for Logotherapy, 32, 84-88.

Harlow, L. L., Newcomb, M. D., \& Bentler, P. M. (1987). Purpose in Life Test assessment using latent variable methods. The British Journal of Clinical Psychology, 26(Pt 3), 235-236. http://dx.doi.org/10.1111/j.2044-8260.1987. tb01355.x. PMid:3664045

Heidegger, M. (1989). Conferências e escritos filosóficos (E. Stein, trad., Coleção Os Pensadores). São Paulo: Nova Cultural.

Hu, L.-T., \& Bentler, P. M. (1999). Cutoff criteria for fit indexes in covariance structure analysis: conventional criteria versus new alternatives. Structural Equation Modeling, 6(1), 1-55. http:// dx.doi.org/10.1080/10705519909540118.

Husserl, E. (1965). A filosofia como ciência de rigor (Trad. A. Beau). Coimbra: Atlantica. (Trabalho original publicado em 1910).

Husserl, E. (2006). Idéias para uma fenomenologia pura e para uma filosofia fenomenológica (M. Suzuki, trad.). Aparecida: Ideias \& Letras. (Trabalho original pubicado em 1913).

Jaspers, K. (1973). Psicopatologia geral. São Paulo: Livraria Atheneu S/A.

Jonsén, E., Fagerström, L., Lundman, B., Nygren, B., Vähäkangas, M., \& Strandberg, G. (2010). Psychometric properties of the Swedish version of the Purpose in Life scale. Scandinavian Journal of Caring Sciences, 24(1), 41-48. http://dx.doi. org/10.1111/j.1471-6712.2008.00682.x. PMid:19889195

Kelloway, E. K. (1998). Using LISREL for structural equationmodeling: a researcher's guide. Thousand Oaks: Sage Publications.

Kierkegaard, S. A. (1976). In vino veritas: la repeticion. Madri: Ediciones Guadarrama.

Kline, R. B. (2010). Principles and practice of structural equation modeling (3rd ed.). New York: Guilford Press.
Längle, A., Orgler, C., \& Kundi, M. (2003). The Existence Scale: a new approach to assess the ability to find personal meaning in life and to reach existential fulfillment. European Psychotherapy, 4, 135-151.

Lukas, E. (1988). Para validar a logoterapia In V. Frankl (Org.), La volundad de sentido (pp. 255-284). Barcelona: Editorial Herder.

Lukas, E. (1989). Logoterapia: "A força desafiadora do espírito" (J. de Sá Porto, trad.). São Paulo: Edições Loyola e Leopoldianum.

Marsh, A., Smith, L., Piek, J., \& Saunders, B. (2003). The Purpose in Life Scale: Psycometric proprieties for social drinkers and drinkers in alcohol treatment. Educational and Psychological Measurement, 63(5), 859-871. http://dx.doi. org/10.1177/0013164403251040.

Marsh, H. W., \& Hocevar, D. (1995). Aplication of confirmatory factor analysis to the study of self-concept: First - and higher-order factor models and their invariance across groups. Psychological Bulletin, 97(3), 562-582. http:// dx.doi.org/10.1037/0033-2909.97.3.562.

Noblejas de la Flor, M. A. (1999). Estructura factorial de los tests PIL y Logotest. Noûs, 3, 67-84.

Nunnally, J. C. (1991). Teoría psicométrica. México: Trillas.

Ortiz, E. M., Buitrago, J. P., \& Osma, J. J. (2010). Validación de la prueba "Escala dimensional de los Recursos Noológicos Aqui y Ahora" para personas mayores de 15 años. Revista Argentina de Clínica Psicológica, 19, 257-272.

Ortiz, E. M., Trujillo, A. M., Castillo, J. P., \& Osma, J. J. (2011). Desarrollo y estructura de la Escala Dimensional del Sentido de Vida. Acta Colombiana de Psicologia, 14, 113-119.

Ortiz, E. M., Cano, A. M. T., \& Trujillo, C. A. (2012). Validación del Test de Propósito Vital (Pil testpurpose in life test) para Colombia. Revista Argentina de Clínica Psicológica, 21, 85-93.

Pasquali, L. (2003). Psicometria: teoria dos testes na psicologia e na educação. Petrópolis: Vozes.

Sartre, J. P. (1987). O Existêncialismo é um Humanismo (R. C. Guedes, trad.). São Paulo: Nova Cultural. (Trabalho original publicado em 1946).

Sartre, J. P. (1997). O ser e o nada: Ensaio de ontologia fenomenológica (5. ed., P. Perdigão, trad.). Petrópolis: Vozes. (Trabalho original pubicado em 1943). 
Thiago Antonio Avellar de Aquino, Valdiney Gouveia Veloso, Andrei Alves de Aguiar, Tiago Deividy Bento Serafim, Alisson de Meneses Pontes, Gylmara de Araújo Pereira \& Ana Sandra Fernandes

Shek, D. (1988). Reliability and factorial structure of the chinese version of the Purpose in Life Questionaire. Psychological Bulletin, 44, 117-118.

Steger, F. M., Frazier, P., Oishi, S., \& Kaler, M. (2006). The meaning in Life Questionnaire: assessing the presence of and search for meaning in life. Journal of Counseling Psychology, 53(1), 80-93. http://dx.doi.org/10.1037/0022-0167.53.1.80.
Steger, M. F. (2009). Meaning in life. In S. J. Lopez (Ed.), Oxford handbook of positive psychology (2nded., pp. 679-687). Oxford: Oxford University Press.

Tabachnick, B. G., \& Fidell, L. S. (1996). Using multivariate statistics (3rd ed.). New York: Harper Collins.

Van de Vijver, F., \& Leung, K. (1997). Methods and data analysis for cross-cultural research Thousand Oaks: Sage Publications. 